\title{
O AUMENTO DA RESISTÊNCIA MECÂNICA DOS NÓS DO BAMBU COM REFORÇO DE FIBRA DE VIDRO
}

\author{
Jorge da Costa Braga ${ }^{1}$ \\ Bárbara Torroglosa Tamasiro ${ }^{2}$ \\ Gabriella Ribeiro Lima ${ }^{3}$ \\ Milena Mendes Furtado de Souza ${ }^{4}$ \\ Paola Jenifer Martins Costa ${ }^{5}$ \\ Pedro Luis Rodrigues Gomes ${ }^{6}$
}

RESUMO: $\mathrm{O}$ aço tem sua empregabilidade consolidada no setor da construção civil devido suas propriedades físicas e mecânicas, contudo, a sua produção causa grandes danos ambientais em razão do alto consumo de energia que resulta no aumento da emissão de gás carbônico das indústrias, a contaminação de rios com efluentes que são emitidos em sua fabricação, e além de tudo isso, não é uma matéria prima renovável. Buscando alternativas que tenham o mínimo impacto ambiental, sem comprometer o resultado conhecido pelo aço, este trabalho foi desenvolvido apresentando uma pesquisa metodológica do bambu Phyllostachys pubescens com o reforço do nó envolvendo-o com a fibra de vidro para aumentar o seu desempenho, podendo ser considerado uma nova alternativa de material para o uso estrutural na engenharia civil. Devido ao meio ambiente ser cada vez mais cobrado pela sociedade e o mercado consumidor, o setor da construção civil não seria diferente. Para estabelecer um parâmetro comparativo que comprove a melhoria da resistência do bambu com o nó reforçado, serão realizados ensaios de compressão, cisalhamento e tração no bambu Phyllostachys pubescens, os CP's foram preparados alguns com tratamento natural (imersão em água), tratamento químico (aplicação de cobre, cromo e boro) e sem tratamento. Por fim, pretende-se comprovar o aumento da resistência mecânica com a aplicação da fibra de vidro do nó do bambu para despertar uma curiosidade nos profissionais brasileiros gerando o aumento em seu uso na construção civil e consequentemente ocorrer a normatização nacional, assim como existem em outros países como China e Colômbia.

Palavras-chave: Bambu. Nó do colmo. Fibra de vidro. Concreto armado. Resistência mecânica.

ABSTRACT: Steel has consolidated employability in the civil construction sector due to its physical and mechanical properties, however, its production causes great environmental damage due to the high energy consumption that results in increased carbon dioxide emissions from industries, river contamination with effluents that are emitted in its manufacture, and besides all that, it is not a renewable raw material. Seeking alternatives that have minimal

\footnotetext{
I Graduação em Engenharia Civil pela Universidade Paulista - UNIP

2. Graduação em Engenharia Civil pela Universidade Paulista - UNIP

3 Graduação em Engenharia Civil pela Universidade Paulista - UNIP

4 Graduação em Engenharia Civil pela Universidade Paulista - UNIP

5 Graduação em Engenharia Civil pela Universidade Paulista - UNIP

6 Graduação em Engenharia Civil pela Universidade Paulista - UNIP
} 
environmental impact, without compromising the result known for steel, this work was developed presenting a methodological research of the bamboo Phyllostachys pubescens with the reinforcement of the knot involving it with fiberglass to increase its performance, which can be considered a new material alternative for structural use in civil engineering. As the environment is increasingly demanded by society and the consumer market, the civil construction sector would be no different. To establish a comparative parameter that proves the improvement in the strength of bamboo with the reinforced knot, compression, shear and traction tests will be carried out on Phyllostachys pubescens bamboo, the CP's were prepared with some natural treatment (immersion in water), chemical treatment (application copper, chromium and boron) and untreated. Finally, it is intended to prove the increase in mechanical strength with the application of fiberglass from the bamboo knot to arouse a curiosity in Brazilian professionals, generating an increase in its use in civil construction and consequently national standardization, as they exist in other countries like China and Colombia.

Keywords: Bamboo. Thatch knot. Fiberglass. Reinforced concrete. Mechanical resistance.

\section{INTRODUÇÃO}

A construção civil está em constante evolução tecnológica, realizando pesquisas sobre a utilização de materiais alternativos para substituição de elementos já consolidados no mercado, com o objetivo de diminuir os impactos ambientais, visando favorecer as gerações futuras, e a redução de custos.

Diante disso, Ghavami (200o) afirma que o bambu é um excelente material para substituir o aço em peças de concreto armado, devido a alta resistência à tração, em relação ao seu peso específico. Por conta de seu excelente desempenho estrutural, sendo leve e ao mesmo tempo resistente, o torna um material viável para se utilizar no lugar do aço. Porém, para o uso estrutural, existem fragilidades, que se originou o interesse ao presente estudo: o reforço do nó do bambu.

Segundo Ghavami et al. (2005), o nó do bambu possui algumas características que prejudicam sua resistência, como a descontinuidade das fibras paralelas em seu nó. Pensando nesta fragilidade, esta pesquisa tem como objetivo utilizar um reforço neste ponto frágil, que segundo Zattera et al. (2000) afirma que a utilização da fibra de vidro eleva a resistência a tração e possui um baixo custo efetivo, corroborado pelo estudo de Fiorelli (2002), demonstrando que o uso das fibras de vidro em construções surpreende com vantagens significativas, tais como: aumento da resistência ao impacto e seu baixo custo.

A espécie de bambu escolhida para o desenvolvimento da pesquisa é a Phyllostachys pubescens, que segundo Ghavami et al. (2003), é uma das espécies com mais resistência à tração, para que se possa substituir completamente o aço em uma viga de concreto armado, como por 
exemplo, em edificações unifamiliares, realizando testes de tração, compressão e cisalhamento no bambu com e sem tratamentos de impermeabilização.

A fundamentação teórica foi dividida em 5 subcapítulos, onde primeiro trata-se da apresentação da estrutura do bambu Phyllostachys pubescens, o segundo apresenta suas principais propriedades, em seguida foi descrito seus possíveis tratamentos químicos e naturais, consecutivamente foi mencionado no $4^{\underline{O}}$ subcapítulo as fragilidades do nó do bambu para seu uso estrutural e por fim foi apresentado as propriedades da fibra de vidro para reforçar o nó.

O artigo completo deve apresentar um limite mínimo de dez (Іо) páginas e um máximo de vinte (20) páginas. As notas técnicas deverão ter no mínimo oito (8) páginas e no máximo quinze (15). Os arquivos para submissão deverão estar em formato Microsoft Word, OpenOffice ou RTF, sem páginas numeradas. Os arquivos não podem ultrapassar $5 \mathrm{Mb}$.

Este documento apresenta a formatação automática do artigo. Serão aceitos trabalhos em português, espanhol e inglês. Não serão aceitos trabalhos de caráter comercial, nem o uso de logotipos de empresas ou de nomes comerciais.

\section{OBJETIVO}

Estudar a aplicabilidade da fibra de vidro com o intuito de melhorar as fragilidades existentes nos nós do bambu, possibilitando sua utilização em concreto armado de estruturas unifamiliares.

\section{Revisão de Literatura}

\section{I Estrutura do bambu Phyllostachys pubescens}

Para a escolha do bambu Phyllostachys pubescens, foram analisadas as propriedades físicas e mecânicas, levando em consideração a sua forma, dimensão e a maneira que as fases são distribuídas no interior do colmo. Passando por etapas de seleção, corte, cura, secagem e tratamentos imunizantes, de forma que seja possível realizar adaptações para melhorar sua performance estrutural.

O bambu é constituído por $40 \%$ de fibras, $50 \%$ de células parenquimosas e $10 \%$ de vasos. De acordo com Ghavami e Rodrigues (2000), a estrutura do bambu é constituídade fibras longas e alinhadas de celulose que são constituintes das paredes celulares das plantas e imersas em uma matriz de lignina cuja função é conferir rigidez, impermeabilidade e resistência a ataques 
microbiológicos e mecânicos aos tecidos vegetais. As fibras do bambu se concentram em maior escala na medida em que a espessura esteja cada vez mais próxima da casca, de forma que o material possa resistiràs ações do vento, que são as cargas mais constantes durante a vida útil do material na natureza. Os vasos do bambu têm como função, distribuir seus nutrientes da raiz às demais partes e, como os colmos são elevados, os vasos são reforçados por um tecido (esclerênquima) que lhes dá resistência.

Para um estudo mais aprofundado sobre a estrutura do bambu, de acordo com Ghavami et al (2017), pode-se dividir em três grupos: macroestrutura, mesoestrutura e microestrutura. $\mathrm{Na}$ macroestrutura, como podemos ver na figura abaixo, o bambu tem umaforma troncocônica, onde o diâmetro na base é maior e, diminui conforme aumenta o comprimento, o colmo tem essa forma pois, a sua base recebe mais solicitações devido a ação do vento e necessita de maior inércia.

Figura 1: Forma tronco-cônica do colmo de bambu
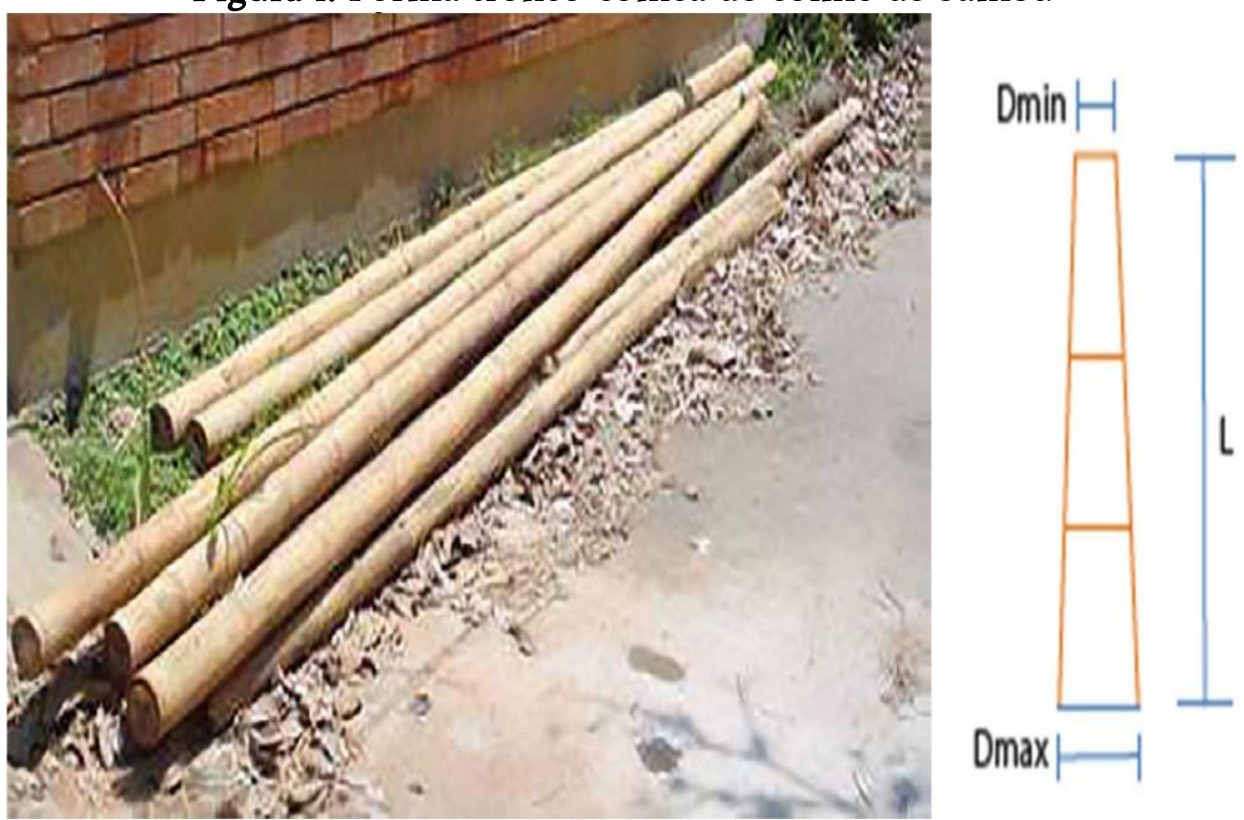

Fonte: Ghavami et al (2017)

Para que o bambu possa ser utilizado em estruturas, é recomendado que o coeficiente de conicidade seja de até $0,67 \%$.

No próximo grupo, segundo Ghavami et al (2017), temos a mesoestrutura do bambu, que é composta de fibras nas direções longitudinais por todo o comprimento do colmo, sofrendo desvios nas regiões dos nós. Na face externa, no sentido radial do colmo aumentam-se a concentração das fibras que são responsáveis pela resistência à tração. 
Figura 2: Desvio do paralelismo das fibras nos nós do colmo

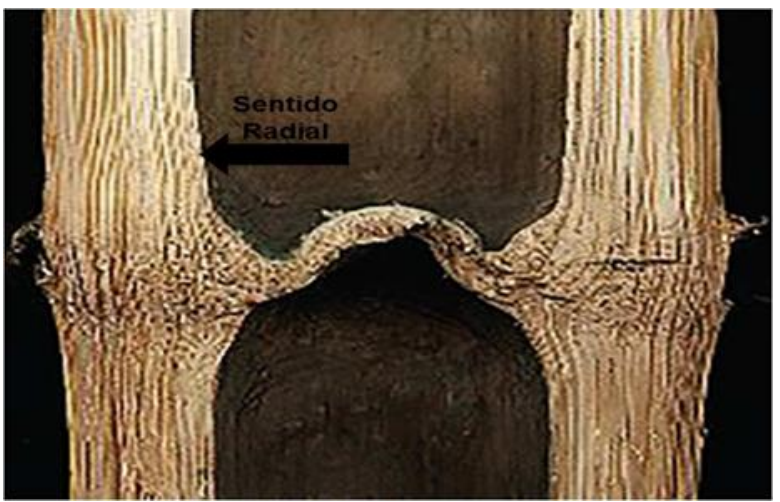

Fonte: Ghavami et al (2017)

Por último, ainda conforme Ghavami et al (2017), a microestrutura do bambu é composta por fibras e vasos que constituem o parênquima, que é um tecido poroso onde se armazena o amido. E por polímeros como a celulose e a lignina, que são responsáveis pela resistência e rigidez, respectivamente.

Figura 3 - Vista microscópica da fibra de bambu

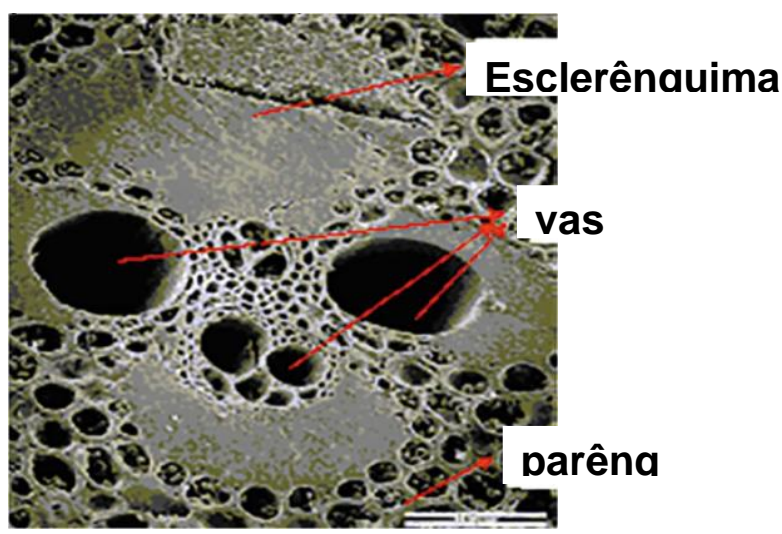

Fonte: Ghavami et al (2017)

\subsection{Propriedades mecânicas do bambu Phyllostachys pubescens}

De acordo com Caeiro (2010), as características estruturais do bambu estãoprincipalmente ligadas ao seu modo de cultivo, os bambus cultivados em terrenos pobres e secos tendem a se apresentarem como mais resistentes quando comparado com outros desenvolvidos em regiões de vales. Sabido isso, é possível observar a importância do cultivo no resultado das propriedades mecânicas do bambu, estas que por sua vez também estaria ligado a outros fatores como, a sua espécie botânica, idade do bambu, umidade, posição dos nós e espessura da parede da planta. 
Conforme cita Barbalho et al (2018) o responsável pela resistência do bambu é o seu alto percentual de fibras ao longo do colmo, e como suas propriedades mecânicas têm grande influência relacionado a umidade externa do ar, faz-se necessário maior precaução com a idade e densidade do bambu na época de colheita.

Ainda segundo Caeiro (2010), divide em cinco as principais propriedades mecânicas do bambu a serem analisadas e levadas em consideração para utilização em construções. São estas: tração, compressão, instabilidade em cargas pontuais, flexibilidade e corte. Todas essas propriedades são responsáveis na seleção do bambu, e por isso devem ser testadas e analisadas através de testes laboratoriais para um uso seguro dentro da construção civil.

Liese (1998) fala que a variação da resistência do colmo é maior na horizontal do que na vertical, e que a densidade dos nós é ainda maior que dos internós, por ter uma quantidade menor de parênquima, porém, é menor sua resistência a tração, compressãoe cisalhamento.

Janssen (2000) cita que a propriedade física que mais influência na propriedade mecânica é a massa específica aparente do material por unidade de volume. $\mathrm{O}$ autor apresenta um estudo comparativo de resistência e dureza de alguns materiais emcomparação à massa específica do bambu, mostrado na Figura 4.

Figura 4 - Resistência e dureza de alguns materiais e o bambu

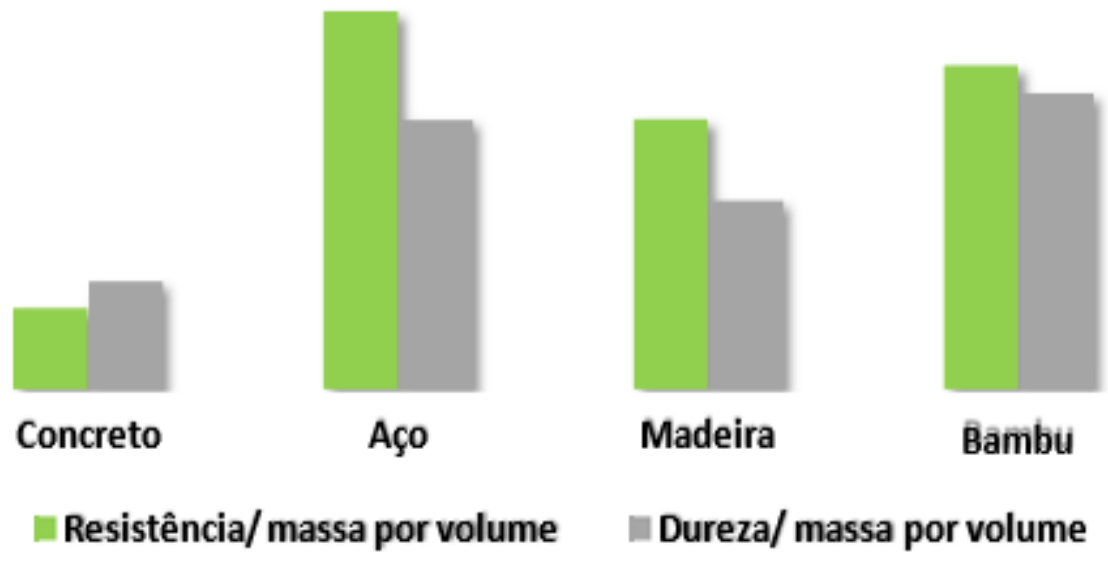

Fonte: Adaptado do Janssen (200o)

Dixon et al.(2014) afirmam que embora o bambu seja botanicamente classificado como da família das gramíneas, devido a sua composição estrutural este muito se aproxima de propriedades físicas e mecânicas de alguns tipos de madeiras utilizadas em construções 
civis com finalidade estrutural, devido a geometria tubular dos colmos, que implica em momentos de inércia similar tanto no eixo $\mathrm{x}$ quanto no $\mathrm{y}$, tornando assim o material de bambu resistente a compressão.

Já os feixes de fibras posicionados de forma paralela na camada intermediária proporcionam alta resistência à tração e às solicitações axiais, enquanto desfavorece a resistência ao cisalhamento, que de acordo com Janssen (1981), é uma importante propriedade dos colmos de bambu, uma vez que pode ser considerada seu ponto fraco

o bambu há um aumento gradativo em sua resistência nos primeiros cinco anos e reduz após os nove anos conforme cita Chun (2003). Conforme avaliação de Li (2004) a densidade aparente do bambu Phyllostachys pubescens em um ano de idade varia da base para o topo entre 0,49 a $0,54 \mathrm{~g} / \mathrm{cm}^{3}$, já aos cinco anos a média fica entre 0,75 a $0,76 \mathrm{~g} / \mathrm{cm}^{3}$, com isso avaliou também o aumento do Modulo de Ruptura (MOR) que apresentouas seguintes variações: em um ano IIo,3 $\mathrm{MPa}$ na base e $117,2 \mathrm{MPa}$ no topo e aos cincoanos $186,2 \mathrm{MPa}$ na base e $183,4 \mathrm{MPa}$ no topo; e o Modulo de Elasticidade (MOE) que também obteve os seguintes resultados: em um ano $7.770 \mathrm{MPa}$ e $8.929 \mathrm{MPa}$ para a base e o topo respectivamente, e aos cinco anos $13.162 \mathrm{MPa}$ e $13.307 \mathrm{MPa}$, chegando assim a valores que aumentam nos primeiros o5 anos de idade do bambu Phyllostachys pubescens em 69\%.

Outras propriedades importantes dos colmos nas construções também são listadas por $\mathrm{Yu}$ (2007), sua composição estrutural e características anatômicas oferece aos colmos elevada resistência a forças axiais, assim como baixa resistência ao cisalhamento, também sua estrutura oca transforma o bambu em um material de alta resistência à flexão.

Segundo Ghavami et al. (2005) a presença de um nó indica a descontinuidade no sentido de algumas fibras na região do nó e mudanças nas seções e nas concentrações das tensões, fazendo do mesmo a região mais frágil para a aplicação do uso estrutural do bambu Phyllostachys pubescens.

O vazio entre os diafragmas chama-se de câmara internodal e a distância entre os nós internos, entrenó ou distância internodal. 


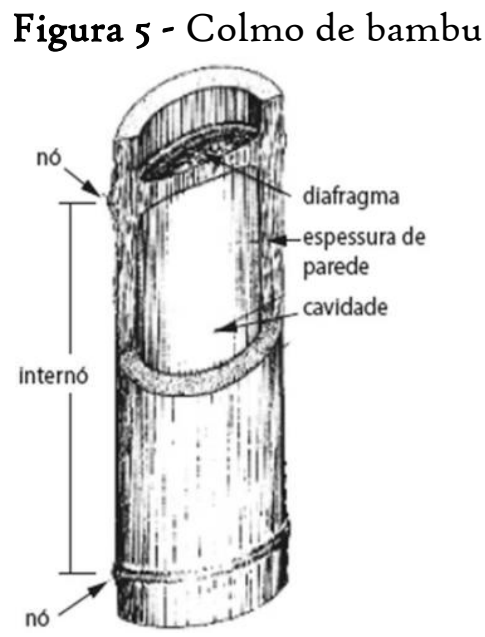

Fonte: Ghavami et al (2017)

\subsection{Tratamentos químicos e naturais para aderência e controle de umidade}

Por ser um material orgânico, segundo Benavides (2019), o bambu Phyllostachys pubescens, sofre com o processo de degradação, principalmente devido ao ataque de fungos, insetos e intemperismo. Essas condições devem ser consideradas com o objetivode amenizar as condições de degradação, eliminando o máximo de fatores externos para prolongar sua durabilidade e preservar suas propriedades mecânicas no emprego de estruturas.

Com isso, se faz necessário aplicação de alguns tratamentos nos colmos colhidos, podendo ser utilizado via natural ou químico. Importante salientar que após a colheita do bambu é fundamental um armazenamento protegido antes de iniciar os tratamentos orientados, conforme indicado por Carbonari et al. (2017).

Figura 6 - Armazenamento correto das varas de bambu

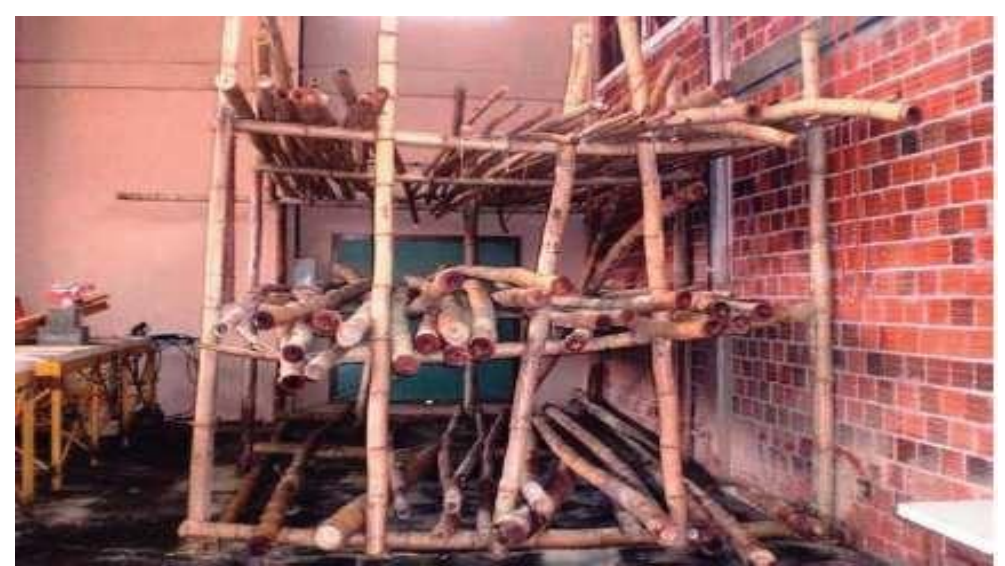

Fonte: Carbonari (2017) 
Um tratamento químico consiste na modificação química da estrutura de um material. Para tratamentos químicos do bambu temos o método de "Boucherie", onde a seiva é retirada através de pressão, e em seu lugar é colocado algum produto químico. Porém, de acordo com Pereira et al. (2008), esse mesmo método tem sua desvantagem, pois com a pressão empregada na aplicação do material pode causar uma ruptura longitudinalno colmo em algumas espécies de bambu.

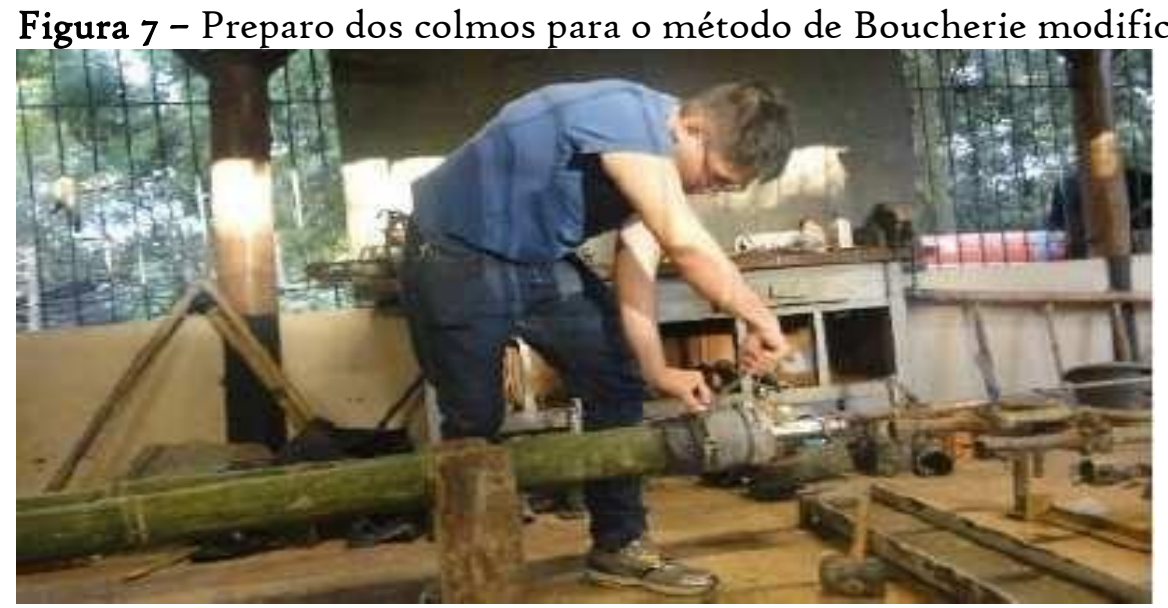

Fonte: Tatibana et al (2016)

De acordo com Neucy (2016) há também o método de transpiração radial onde o bambu é posto na vertical sobre um tambor com um produto químico aquecido para se transformar em vapor.

Para Tatibana et al (2016) outra forma de tratamento é por imersão, onde o bambu é imerso em uma substância química (resina epóxi) que preencha os vazios presentes na microestrutura do bambu. $O$ tratamento com resina epóxi para o preenchimento de vazios presentes no colmoprevine o ataque de insetos Dinoderus minutus, consequentemente aumentando a durabilidade do bambu além de amplificar sua resistência à tração, compressão e flexão.

Conforme Tiburtino et al. (2015) dos métodos de tratamento químico, o mais indicado para prevenção da saúde dos operadores que realizarão esse procedimento e ao meio ambiente é a aplicação de produtos à base de cobre, cromo e boro (CCB). Essa classificação ocorre pela ausência do Arsênico, altamente prejudicial à saúde. 
Figura 8 - Broca do bambu ou caruncho dentro do bambu

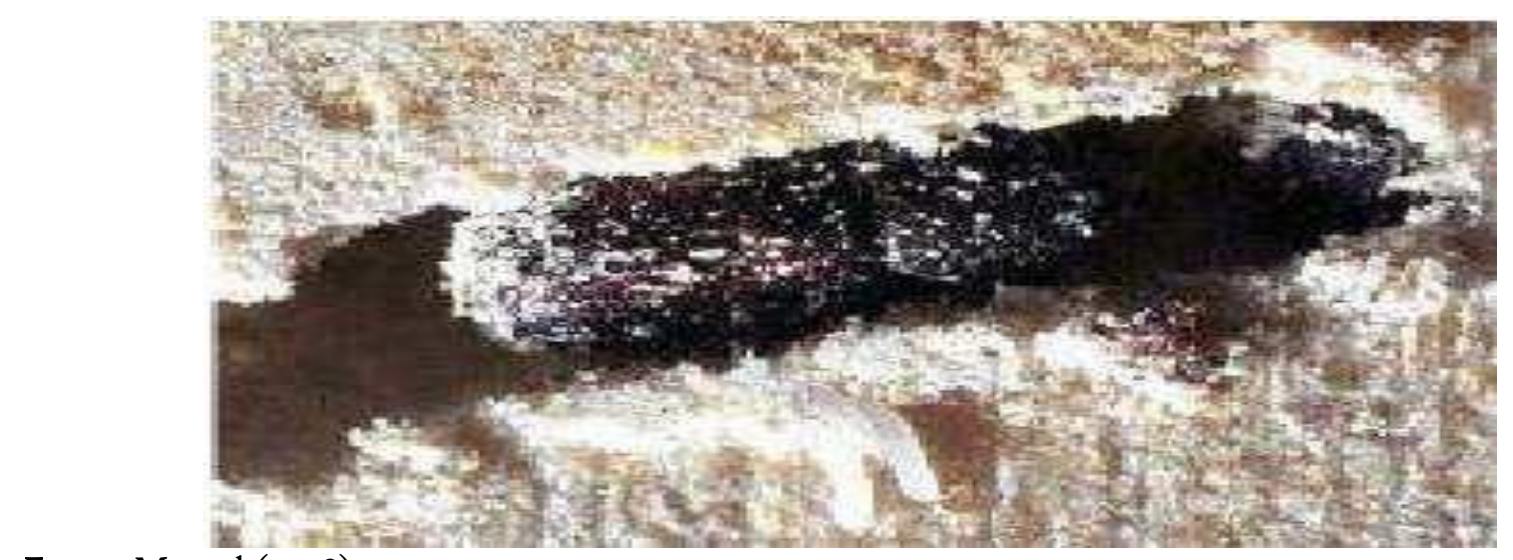

Fonte: Marçal (2008)

Os tratamentos naturais adotados podem ser através do fogo, da água e na própria mata após a extração da planta. No tratamento na mata, o bambu é posicionado na vertical para evitar o contato com o solo, evitando o ataque de brocas (pragas que se alimentam de madeiras). No tratamento com o fogo, o bambu Phyllostachys pubescens é exposto àqueima que tem como finalidade desfazer do amido que atrai fungos e insetos, assim como o tratamento com água onde o bambu é deixado submerso por semanas.

Figura 9 - Tratamento natural pelo método de imersão

Fonte: Teixeira (2006)

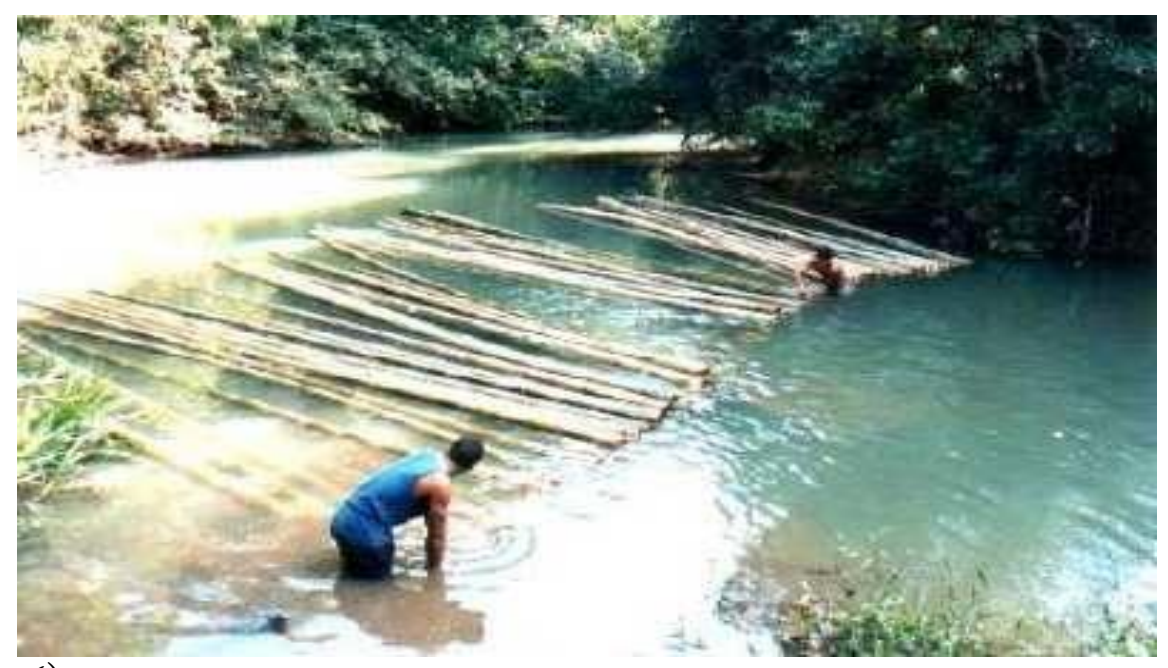

\subsection{Fragilidade nos nós do bambu Phyllostachys pubescens para uso estrutural}

De acordo com Ghavami e Hombeeck (198I) foi observado que uma grande parte das rupturas do bambu acontece nos nós. Isso ocorre devido, a desorganização na disposição das fibras na região dos nós, que é o oposto do que acontece nos entrenós onde 
temos as fibras paralelas e ordenadas ao eixo.

Segundo Liese (1998), as características morfológicas do nó são dadas pelo diafragma que pode ser reto, côncavo ou convexo e pelo intranó, formado pela cicatriz da bainha e da aresta nodal (protuberâncias) do nó, e por serem pontos fracos, é onde a camada superficial de sílica se interrompe e o colmo é mais indefeso.

$\mathrm{Na}$ figura ıo, é possível identificar que existe uma maior concentração de fibras no sentido radial do colmo permitindo uma maior resistência.

Figura 1o - Imagem microscópica evidenciando a concentração de fibras
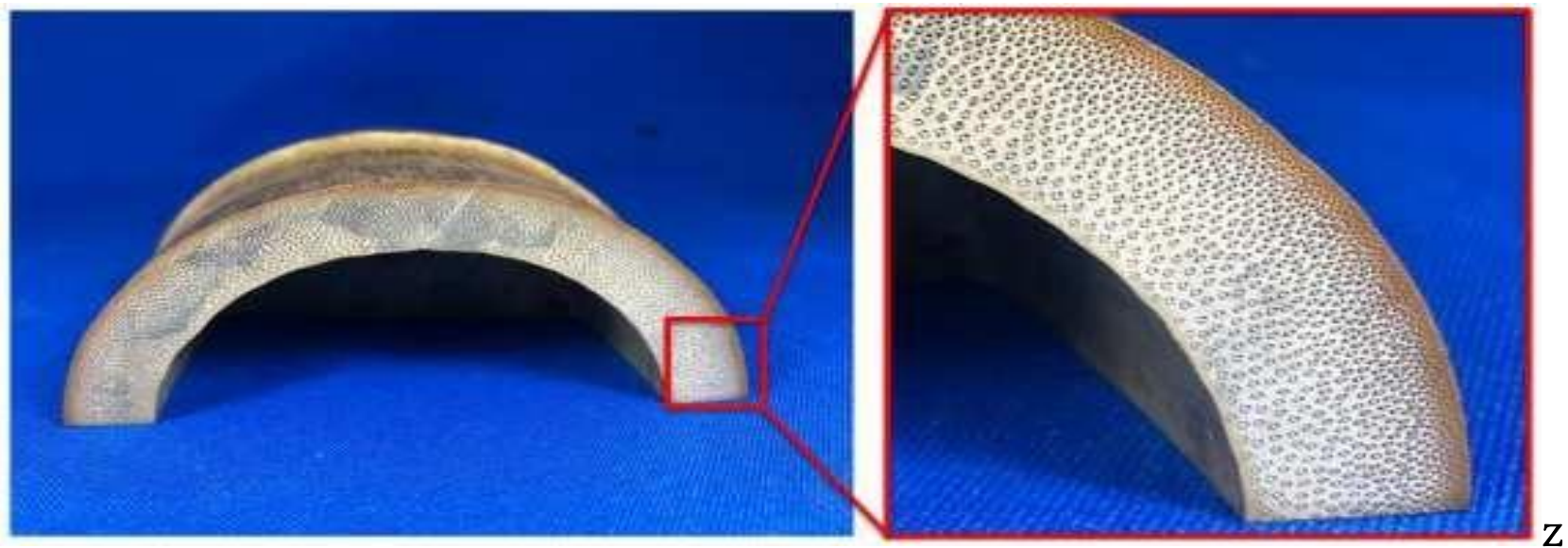

Fonte: Gomes (2019)

Totalmente diferente do que acontece nos nós, de acordo com a figura abaixo ilustrado por Culzoni (1986), comprovando o ponto de fragilidade do bambu: o nó.

Figura II - Imagem microscópica evidenciando a descontinuidade das fibras no nó
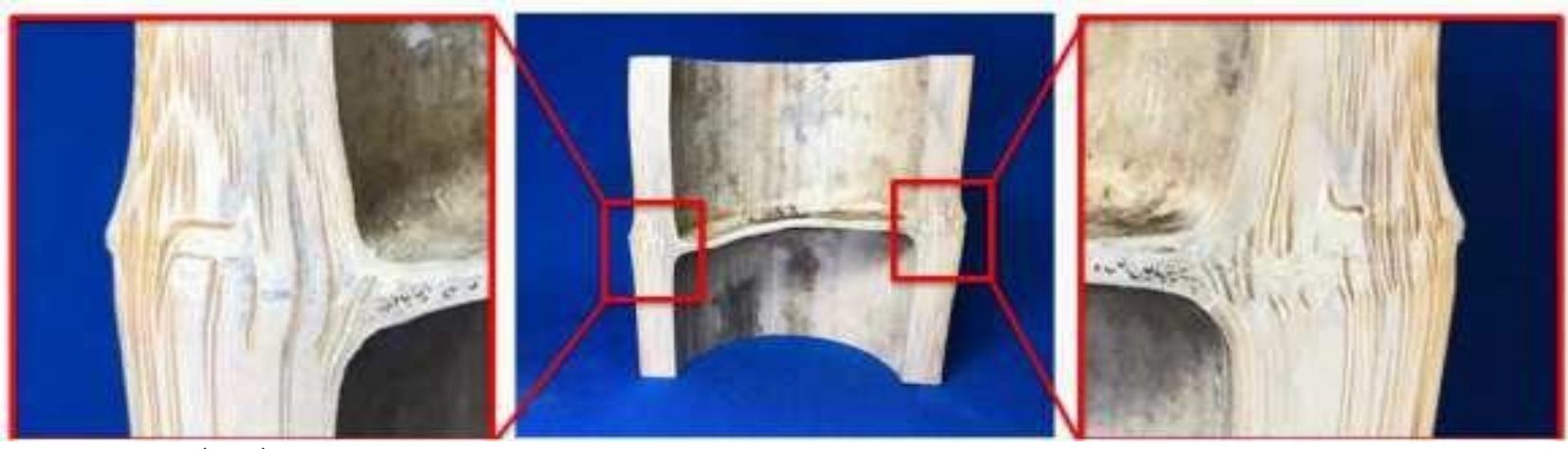

Fonte: Gomes (2019)

Lopez (1974) foi um dos primeiros estudiosos a mergulharem nessa possibilidade de 
estudo do bambu, realizando testes para comprovação de sua resistência. Para isso, ele utilizou corpos de prova de $30 \mathrm{~cm}$ de comprimento e $3 \mathrm{~cm}$ de diâmetro, chegando ao resultado de $80 \mathrm{MPa}$. Após os testes realizados ele demonstrou que sim, era possível utilizar o bambu como reforço no concreto, pois a curva de tensão-deformação secomportou na medida esperada entre $20 \mathrm{MPa}$ e I20 MPa.

Devido a descontinuidade natural das fibras na região do nó do bambu ocorre seu rompimento quando solicitado a resistência a tração, conforme apresenta ensaios realizados por Ghavami e Marinho (2005), a resistência a tração atingiu um valor médio de 89,96 MPa, sendo que os corpos de prova que continham nó apresentaram as menores resistências.

Após ensaios realizados por Carbonari et al (2017), foi observado a diminuição da resistência nos CPs, constituídos por parte interna da parede do bambu, além de tambémreduzir significantemente a resistência à tração, proporcionando uma ruptura frágil e abrupta, o que não ocorre nas áreas do colmo sem presença de nós, nos quais vão se rompendo fibra por fibra, apresentando maior ductilidade.

Figura 12 - Rompimento de corpo de prova de bambu por compressão axial.

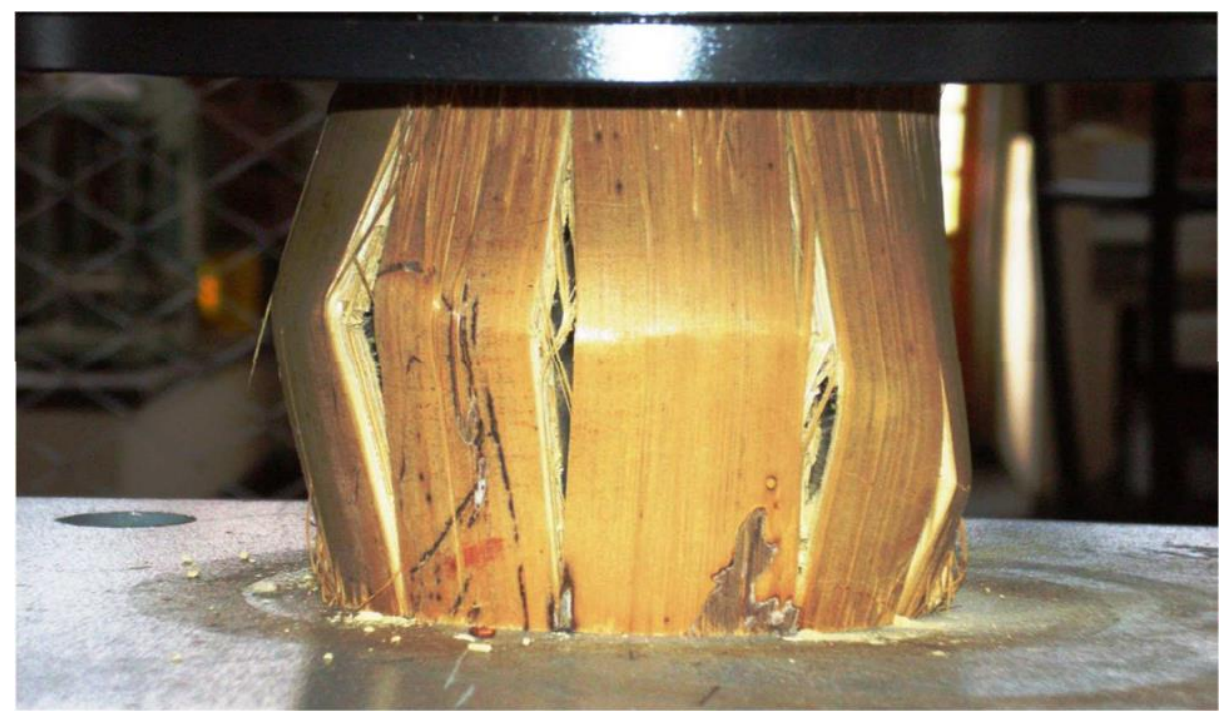

Fonte: Carbonari et al (2017)

\subsection{O reforço com fibra de vidro nos nós do bambu Phyllostachys pubescens}

Como demonstrado nos tópicos anteriores e comprovado por testes do estudioso Ghavami (2005), a fragilidade do bambu se encontra no nó.

De acordo com estudos realizados por Ota (2004), a confecção de fibras de vidro iniciou 
na Antiga Síria, Grécia e Egito. No decorrer da 2o Guerra Mundial, com o objetivo de aumentar a rigidez dos equipamentos bélicos, iniciou-se a comercialização da fibra devidro como item de reforço das armaduras e equipamentos dos soldados.

As fibras em sua composição possuem vários elementos químicos e, por ser um material fluído, ele se adapta melhor às cargas dinâmicas. Entretanto, conforme cita Kemerich et al. (2013) nada mais é, do queum composto de matriz de resina plástica reforçada com fibras de vidro.

Independentemente de sua composição, Oliveira (1995) demonstrou em seu estudo que o uso da fibra de vidro aumenta a competência do objeto em suportar cargasmaiores, em especial quando sujeitas ao esforço de flexo-tração, tração e impacto. E Fiorelli (2002) apresenta um estudo demonstrando que o uso das fibras de vidro em construções surpreende com vantagens significativas, tais como: aumento da resistênciaao impacto e seu baixo custo.

Se aprofundando na vantagem térmica, Zattera et al (200o) realizou testes onde notou possível comprovar que a fibra de vidro possui alta resistência térmica, podendo reter de $37 \mathrm{I}^{\circ} \mathrm{C}$ até $537^{\circ} \mathrm{C}$. Possui também a alta condutividade térmica, e baixo coeficiente de expansão térmica. É também um material de alta resistência dielétrica e, incombustível que não se propaga, nem desencadeia chamas.

Também afirma que as propriedades das fibras de vidro são capazes de aprimoraro desempenho dos materiais aos quais se encontram atrelados. Além disso, a fibra de vidro possui custo efetivo reduzido, ao se analisar as possibilidades oferecidas pelo material, em comparação com outros materiais sintéticos e naturais.

E por fim, para Zattera et al (2000), a fibra de vidro possui as seguintes propriedades: Em relação à sua resistência mecânica, seus filamentos possuem alta resistência à tração, tanto quanto a do fio de aço. Existe também uma boa resistência química, podendo ser exposta a condições de umidade excessiva, insalubridade e afins. Há também estabilidade dimensional, pois sofre menores variações mediante o aquecimento ou arrefecimento

De acordo com Carvalho (1992), as fibras de vidros apresentam excelente aderência fibra/matriz, devido ao tratamento que as mesmas, recebem (denominado de encimagem) para serem utilizadas junto às matrizes orgânicas, possuem boas propriedades elétricas e grandes vantagens no que diz respeito à aplicação e custo. 
Figura 13 - Fibra de vidro

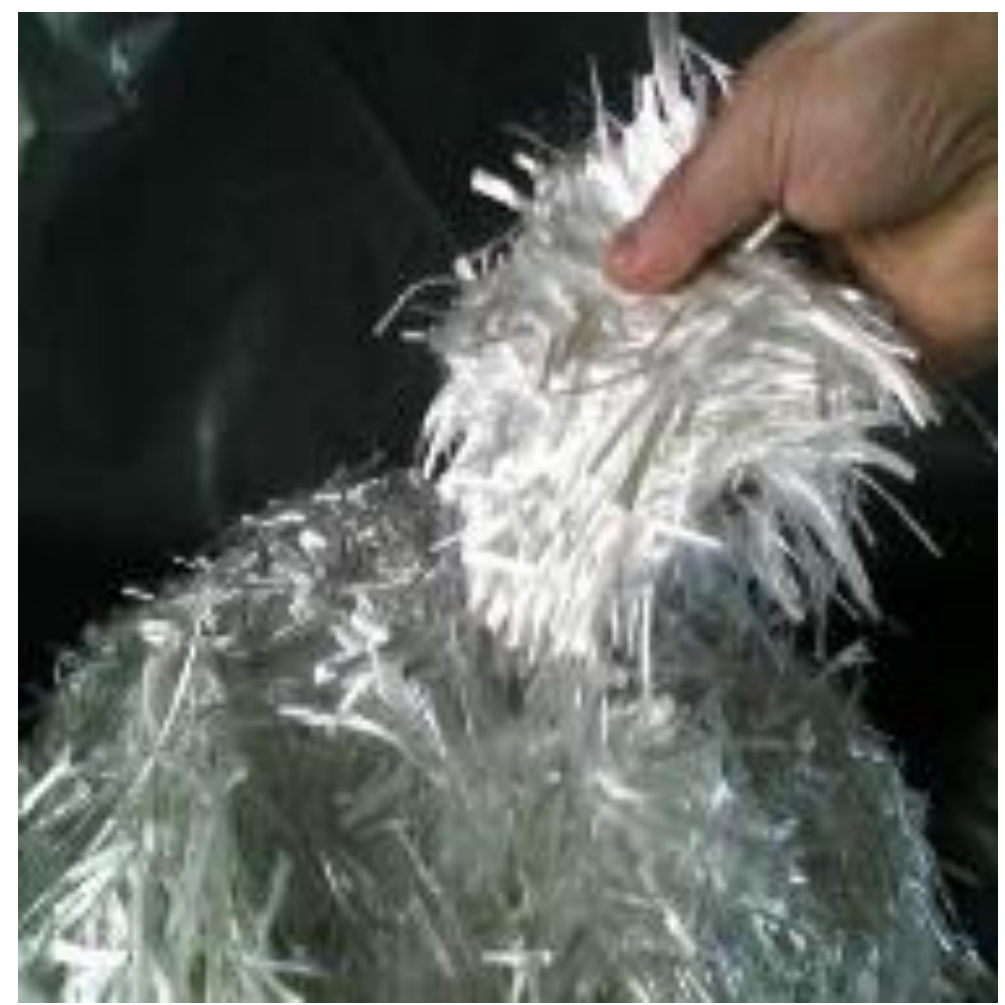

Fibertex - catalogo (2012)

Conforme Tinô (2010), o tipo mais usado industrialmente é a fibra de vidro do tipo E, a qual se destaca, por possuir baixo teor de álcali e excelentes propriedades elétricas, isolantes, além de boas propriedades de resistência mecânica, rigidez e desgaste.

Oliveira (2012), cita como algumas das vantagens de se utilizar fibra de vidro a facilidade que é estirado em fibras de alta resistência a partir do seu estado fundido, material que é facilmente disponível e pode ser fabricado economicamente em um plásticoreforçado com vidro, com ampla variedade de técnicas para fabricação. É relativamente resistente, principalmente quando incorporado à uma matriz de plástico, aumentando a resistência específica do compósito e possuir uma inércia química tornando o compósito conveniente para ambientes corrosivos.

\section{METODOLOGIA}

Tatibana R. M, et al. (2016) afirma que para o uso do bambu como reforço em estruturas de concreto armado, é necessário que os coeficientes de segurança sejam mais conservadores do que os estipulados na NBR. 
Andrea, et al. (2019) concluiu em sua pesquisa que, para aplicar o bambu estruturalmente é necessário considerar as propriedades físicas dos colmos, sua espessura das paredes e sua densidade além de, realizar por meio de inspeção visual e a termografia, meios recomendados devidos causarem menor impacto no material.

Greice Hellen, et al. (2019) confirma a aplicabilidade do bambu em colunas, pilares e peças estruturais onde têm esforços solicitantes de compressão axial, através do estudo da planta em conjunto aos ensaios de compressão axial foi obtido resultados onde a resistência atingida se enquadra nas mínimas que foram normatizadas para utilização do concreto, sendo este $25 \mathrm{MPa}$.

Em estudos realizados por Carbonari, et al. (2017) foi observado que o bambu apresenta valores eficientes de resistência mecânica de tração e compressão em relação ao aço e ao concreto e, alta flexibilidade, vantagem em relação a ações de vento mesmo sendo considerado um material leve.

Em estudos realizados por Gibson, et al. (2014). mostram que existe uma variação significante entre as propriedades mecânicas dependendo da posição radial, fator determinante para tornar os requisitos de rigidez e peso limitantes para sua aplicação estrutural.

Estudos realizados por Ghavami, et al. (2005) mostraram que, o bambu possui alta resistência à tração, sendo 50\% superior a sua resistência à compressão sendo superior ainda, em regiões onde não há presença de nó.

O módulo de resistência varia ao longo do colmo devido seu diâmetro e espessura e, que sua resistência média à tração apresenta maiores resultados na parte central chegando à 95,80 $\mathrm{MPa}$ na região sem nó e 82,6icom nó. A resistência à compressão é maior na parte superior do colmo, com resultado experimental de 34,52 sem nó e, 29,62 MPa com nó.

A fim de aumentar a durabilidade e eficiência das propriedades mecânicas do bambu Ghavami, et al. (2017), com uso de resinas poliméricas preencheu os vazios dos vasos condutores de seiva do bambu.

\section{RESULTADOS}

Com o intuito de encontrar alternativas econômicas e reduzir o impacto ambiental causado pela produção do aço, foi estudado materiais que possibilitem sua substituição no concreto armado procurando manter suas características mecânicas e eliminando uma de suas principais deficiências como a corrosão. Entre vários materiais, o bambu se destacou em nossas pesquisas, pois ele vem sendo estudado em nosso país como um substituto a altura do aço, 
assim como já utilizado em alguns países como China e Colômbia que possuem normas técnicas desse material.

Embora várias espécies de bambu já estão implantadas no cotidiano de pesquisas nacionais e uso internacional, pouco se fala de sua fragilidade do nó e de soluções para essa patologia. Com isso, neste estudo, após demonstrar a fragilidade do nó do bambu Phyllostachys pubescens, será analisado a possibilidade de envolver o nó do bambu comuma camada de fibra de vidro com o objetivo de aumentar sua resistência e eliminar essa fragilidade demonstrando ainda mais eficácia na substituição do aço.

\section{CONCLUSÃO}

O uso do bambu tem sido implantado em diversos locais no mundo, mas no Brasilainda é uma ideia pouco disseminada e aplicada, devido a descriminação que existe em nosso país, pois este material é utilizado em sua maior parte em áreas subdesenvolvidas.

Devido à falta de normatização nacional, poucos profissionais conhecem as características deste material, o que acabou dificultando nossas pesquisas por ter poucos autores nacionais que discorram sobre o tema, expondo suas vantagens da aplicação naconstrução civil na realidade do nosso país, além disso, outro desafio foi determinar a espécie do bambu a ser utilizado.

Após comprovado a resistência do bambu Phyllostachys pubescens conforme exposto na pesquisa bibliográfica, foi identificado que há uma fragilidade no nó, e com o intuito de eliminar esta deficiência, analisamos a aplicabilidade de uma luva de fibra de vidro para reforçar o nó aumentando assim a sua resistência.

Com isso, o intuito deste trabalho é instigar a sociedade da construção civil, a pesquisar e utilizar este material renovável, trazendo benefícios ao meio ambiente devido à redução de emissão de gás carbônico em sua produção, consequentemente reduzindo o impacto do efeito estufa. Sendo um material de baixo custo e de fácil aquisição e manuseio, traz uma boa relação de custo benefício para implantação na construção civil. 


\section{REFERÊNCIAS BIBLIOGRÁFICAS}

BARBALHO, GREICE HELLEN DE NOVAES; FERREIRA, MAURÍCIO CAMARGO. BAMBU: AÇO VERDE DA ARQUITETURA. UNIMONTE, 2019. DISPONÍVEL EM

: $\quad$ HTTP://WWWi.SP.SENAC.BR/HOTSITES/BLOGS/REVISTAINICIACAO/WPCONTENT/UPLOADS/2019/03/246_IC_ARTIGOREVISADO-2.PDF ACESSO EM: ABR. 2021.

BARBALHO, G. H. N.; GARCIA, D. V.; SILVA, J. R. APLICAÇÃO DO MATLAB EM ANÁLISE DE RESISTÊNCIA MECÂNICA DE COMPRESSÃO AXIAL DO BAMBU NO CONCRETO. REVISTA UNISANTA CIÊNCIA E TECNOLOGIA, VOL. 7 № 2, V. 17 , P. 56-62, 2018. DISPONÍVEL EM: HTTPS://PERIODICOS.UNISANTA.BR/INDEX.PHP/SAT. ACESSO EM: ABR. 202I.

BENAVIDES, A. J.; FERENHOF, H.; VAlle, A.; LIBRElotTO, L. ENSAIOS POTENCIAIS

PARA AVALIAÇÃO DA DURABILIDADE DO BAMBU EM EDIFICAÇÕES: BUSCA SISTEMÁTICA. UFSC, MIX SUSTENTÁVEL, $\quad$ V. $\quad 5, \quad \mathrm{~N} . \quad 3, \quad 2019$. DISPONÍVEL

EM:

HTTPS://OJS.SITES.UFSC.BR/INDEX.PHP/MIXSUSTENTAVEL/ARTICLE/VIEW/3 397/o. ACESSO EM: ABR. 2021.

BONIFÁCIO, J. S. R.; GODINHO, D. S. S. ESTUDO DO EFEITO DAS FIBRAS DE VIDRO E POLIPROPILENO NAS PROPRIEDADES MECÂNICAS DO CONCRETO. UNIVERSIDADE DO EXTREMO SUL CATARINENSE， 20I4. DISPONÍVEL

EM:

HTTP://REPOSITORIO.UNESC.NET/BITSTREAM/r/3028/ז/J\%C3\%A9SSICASPRICIG OROCHABONIF\%C3\% AICIO.PDF. ACESSO EM: ABR. 2021.

BRAGA NETO, JOSÉ LIRA. ESTUDO DO COMPORTAMENTO MECÂNICO DE COMPÓSITOS DE MATRIZ POLIMÉRICA COM FIBRA DE VIDRO CONTENDO DESCONTINUIDADES GEOMÉTRICAS. UNIVERSIDADE FEDERAL DE CAMPINA GRANDE, 2015.

PPGEM.UFCG.EDU.BR/ARQUIVOS/2015/JOS\%C3\%89\%20LIRA\%20BRAGA\%20NETO \%202015.PDF. ACESSO EM: ABR. 2021.

CAEIRO, JOÃO GABRIEL DE MATOS. CONSTRUÇÃO EM BAMBU. UNIVERSIDADE DE LISBOA, zoro. DISPONÍVEL EM: 
HTTPS://WWW.REPOSITORY.UTL.PT/HANDLE/I040o.5/3337. ACESSO EM: ABR. 2021.

CAPElla, M. C.; SOUfEN, C. A.; CORREIA, G. V.; GRIZOLA, O. S.; PINTÃO, C. A. F.;

IMAIZUMI, M. PROPRIEDADES MECÂNICAS EM LAMINADOS FIBRAS DE VIDRO E FIBRA DE CARBONO EM RESINA EPÓXI. 20으르IMAT - CONGRESSO BRASILEIRO DE ENGENHARIA E CIÊNCIA DOS MATERIAIS, 20I2. DISPONÍVEL EM: HTTPS://DOCPLAYER.COM.BR/ro821970-PROPRIEDADES- MECANICAS-EMLAMINADOS-FIBRAS-DE-VIDRO-E-FIBRA-DE-CARBONO-EM-RESINAEPOXI.HTML.

ACESSO EM: ABR. 202I.

CARNOBARI, G.; JUNIOR, N. M. S.; PEDROSA, N. M.; ABE, C. H.; SCHOLTZ, M. F.; ACOSTA, C. C. V.; SMITH, S. E.; CARBONARI, L. T. REVISTA MIX SUSTENTÁVEL, V. 03,

P. 17-25, 2017. DISPONÍVEL EM:

HTTPS://OJS.SITES.UFSC.BR/INDEX.PHP/MIXSUSTENTAVEL/ARTICLE/VIEW/I 876 ACESSO EM: ABR. 2021.

CARVALHO, A.: "FIBERGLASS X CORROSÃO - ESPECIFICAÇÃO, INSTALAÇÃO E MANUTENÇÃO DE EQUIPAMENTOS DE FIBERGLASS PARA AMBIENTES AGRESSIVOS”, ASPLAR, 425 P., SÃO PAULO, 1992. DISPONÍVEL EM:

HTTPS://PPGEM.UFCG.EDU.BR/ARQUIVOS/2015/JOS\%C3\%89\%20LIRA\%2oBRAGA\% 2оNETO

\%202015.PDF. ACESSO EM: ABR. 2021.

DIXON, P. G.; GIBSON, L. J. THE STRUCTURE AND MECHANICS OF MOSO BAMBOO MATERIAL. JOURNAL OF THE ROYAL SOCIETY INTERFACE, V. II, P. II2, 2014. DOI: HTTPS://DOI.ORG/I0.1098/RSIF.2014.0321. ACESSO EM: ABR. 2021.

FIORELLI, JULIANO. UTILIZAÇÃO DE FIBRAS DE CARBONO E DE FIBRAS DE VIDRO PARA REFORÇO DE VIGAS DE MADEIRA. UNIVERSIDADE D /88/88131/TDE-I2052003- I44536/PUBLICO/DISJULIANO.PDF. ACESSO EM: ABR. 2021.

GHAVAMI, KHOSROW. BAMBOO AS REINFORCEMENT IN STRUCTURAL CONCRETE ELEMENTS. CEMENT \& CONCRETE COMPOSITES 27 (2005) 637-649. DISPONÍVEL EM: HTTPS://WWW.RESEARCHGATE.NET/PUBLICATION/22268472I_BAMBOO_AS_RE INFORCEMENT_IN_STRU CTURAL_CONCRETE_ELEMENTS. ACESSO EM: ABR. 2021. 
GHAVAMI, K.; CULZONI, R. A. M. UTILIZAÇÃO DO BAMBU COMO MATERIAL EM HABITAÇÃO DE BAIXO CUSTO. INTERNATIONAL SYMPOSIUM ON TRANSFER AND PRODUCTION OF HOUSING TECHNOLOGY IN RESEARCH AND PRATICE HABITEC 87, 1987, SÃO PAULO. PROCEEDINGS...SÃO PAULO: IPT AND CIB, 1987. P.I8I-188.

GHAVAMI, K.; MARINHO, A. B. PROPRIEDADES FÍSICAS E MECÂNICAS DO COLMO INTEIRO DO BAMBU DA ESPÉCIE GUADUA ANGUSTIFOLIA. REVISTA BRASILEIRA DE ENGENHARIA AGRÍCOLA E AMBIENTAL, V.9, N.I, P.Io7-II4,

\section{ACESSO EM: ABR. 2021.}

GHAVAMI, K.; BARBOSA, N. P.; MOREIRA, L. E. BAMBU COMO MATERIAL DE ENGENHARIA. AVALIAÇÃO DE DESEMPENHO DE TECNOLOGIAS CONSTRUTIVAS INOVADORAS: CONFORTO AMBIENTAL, DURABILIDADE E PÓS-OCUPAÇÃO (PP.305-348). DISPONÍVEL EM: HTTPS://WWW.RESEARCHGATE.NET/PUBLICATION/320208925_BAMBU_COMO_ MATERIAL_DE_ENGEN HARIA. ACESSO EM: ABR. 2021.

GHAVAMI, K.; HOMBEECK, R.V. APPLICATION OF BAMBOO AS A CONSTRUCTION MATERIAL. PART I- MECHANICAL PROPERTIES \& WATER REPELLENT TREATMENT OF BAMBOO, PART II- BAMBOO REINFORCED CONCRETE BEAMS. LATIN AMERICAN SYMPOSIUM ON RATIONAL ORGANIZATION OF BUILDING APPLIED TO LOW COST HOUSING, I98I, SÃO PAULO. PROCEEDINGS...SÃO PAULO: IPT/CIB, I98ı. P. 49-66, V.ı.

GHAVAMI, K.; RODRIGUES, C.S. ENGINEERING MATERIALS AND COMPONENTS WITH PLANTS. CIB-SYMPOSIUM, CONSTRUCTION \& ENVIRONMENT, 2000, SÃO PAULO. PROCEEDINGS...SÃO PAULO: GLOBAL SEVEN EDITOR, 2000.

GOMES, BRUNO MENEZES DA CUNHA. CARACTERIZAÇÃO MECÂNICA, ESTABILIDADE TÉRMICA E REAÇÃO AO FOGO DO BAMBU MOSSÔ (L. PHYLLOSTACHYS PUBESCENS). UNIVERSIDADE FEDERAL DO RIO DE JANEIRO, 2019. DISPONÍVEL EM: HTTPS://PANTHEON.UFRJ.BR/HANDLE/11422/13692. ACESSO EM: ABR. 2021.

JANSSEN, J. J. A.; PROJETANDO E CONSTRUINDO COM COM BAMBU. COCOLANDIA, $\mathrm{V}$. 20, P. 22-26, 2000. DISPONÍVEL EM: HTTP://COCOLANDIA.ORG/RESOURCES/BUILDING_WITH_BAMBOO.PDF. ACESSO EM: ABR. 202I.

JANSSEN, J. J. A. DESIGNING AND BUILDING WITH BAMBOO. (TECHNICAL REPORT; VOL. 20). INTERNATIONAL NETWORK FOR BAMBOO AND RATTAN, 2000. 
HTTPS://RESEARCH.TUE.NL/EN/PUBLICATIONS/DESIGNING-AND-BUILDINGWITH-BAMBOO-2. ACESSO EM: ABR. 2021.

LIESE, WALTER. THE ANATOMY OF BAMBOO CULMS. INTERNATIONAL NETWORK FOR BAMBOO AND RATTAN, 1998. DISPONÍVEL EM: HTTPS://WWW.INBAR.INT/WP-CONTENT/UPLOADS/2017/o3/THE-ANATOMYOF-BAMBOO-CULMS_1998.PDF. ACESSO EM: ABR. 2021.

LOPÉZ, O. H. BAMBU, SU CULTIVO Y APLICACIONES EN FABRICACCIÓN DE PAPEL, CONSTRUCCIÓN, ARQUITECTURA, INGENIERIA, ARTESANIA. CALI, COLOMBIA: ESTUDIOS TECNICOS COLOMBIANOS LTDA, 1974.

MARÇAL, VITOR HUGO SILVA. USO DO BAMBU NA CONSTRUÇÃO CIVIL. UNIVERSIDADE DE BRASÍLIA, 2008. DISPONÍVEL EM: C. ACESSO EM: ABR. 2021.

OLIVEIRA, CLAITON SOMMARIVA; VITO, MÁRCIO. SUBSTITUIÇÃO TOTAL DO AÇO, USANDO BAMBU COMO ARMADURA DE COMBATE A FLEXÃO EM VIGAS DE CONCRETO. REPOSITÓRIO UNIVERSIDADE EXTREMO SUL CATARINENSE, 2012. DISPONÍVEL EM: HTTP://REPOSITORIO.UNESC.NET/BITSTREAM/I/ı535/I/CLAITON\%2oSOMMARI VA\%2oDE\%20OLIVEIR A.PDF. ACESSO EM: ABR. 2021.

OLIVEIRA, LUIZ FERNANDO ANDRADE. CONHECENDO BAMBUS E SUAS POTENCIALIDADES PARA USO NA CONSTRUÇÃO CIVIL. UNIVERSIDADE FEDERAL DE MINAS GERAIS, 2013. DISPONÍVEL EM: HTTPS://REPOSITORIO.UFMG.BR/BITSTREAM/r843/BUBD-

9GBPWL/I/MONOGRAFIA_CONHECENDO_BAMBUS_E_SUAS_POTENCIALIDADE S LUIZ_FERNANDO

_ANDRADE DE_OLIVEIRA.PDF. ACESSO EM: ABR. 2021.

PERUZZI, ANTÔNIO DE PAULO. COMPORTAMENTO DAS FIBRAS DE VIDRO CONVENCIONAIS EM MATRIZ DE CIMENTO PORTLAND MODIFICADA COM LÁTEX E ADIÇÃO DE SÍlICA ATIVA. UNIVERSIDADE DE SÃO PAULO, 2002. DISPONÍVEL EM:

HTTPS://WWW.TESES.USP.BR/TESES/DISPONIVEIS/18/18131/TDE-13112002I806ı/PUBLICO/ANTONIOPPERUZZI.PDF. ACESSO EM: ABR. 2021.

TATIBANA, R. M.; REIS, M. P.; BIANCHI, G. BAMBU COMO MATÉRIA-PRIMA PARA CONSTRUÇÕES SUSTENTÁVEIS. REVISTA PERIÓDICO TÉCNICO E CIENTÍFICO CIDADES VERDES, V. 04, P. 95-103, 2016. DISPONÍVEL EM:

HTTPS://WWW.AMIGOSDANATUREZA.ORG.BR/PUBLICACOES/INDEX.PHP/CI DADES_VERDES/ARTICLE/DO WNLOAD/I394/I416. ACESSO EM: MAR. 2021. 
TEIXEIRA, ANELIZABETE ALVES. PAINÉIS DE BAMBU PARA HABITAÇÕES ECONÔMICAS: AVALIAÇÃO DO DESEMPENHO DE PANÉIS REVESTIDOS COM ARGAMASSA. UNIVERSIDADE DE BRASÍLIA, 2006. DISPONÍVEL EM: HTTPS://REPOSITORIO.UNB.BR/HANDLE/ro482/5185. ACESSO EM: ABR. 202I.

TINÔ, SÉRGIO RENAN LOPES. DESCONTINUIDADE NA SEÇÃO TRANSVERSAL EM LAMINADOS COMPÓSITOS POLIMÉRICOS: EFEITOS E PROPRIEDADES. UNIVERSIDADE FEDERAL DO RIO GRANDE DONORTE， 2oro. DISPONÍVEL EM:

HTTPS://REPOSITORIO.UFRN.BR/BITSTREAM/123456789/15606/I/SERGIORLT_DISE RT.PDF. ACESSO EM: ABR. 2021.

YU, XIAOBING. BAMBOO: STRUCTURE AND CULTURE. UNIVERSITÄT DUISBURG-ESSEN, 2007. DISPONÍVEL EM: HTTPS://CORE.AC.UK/DOWNLOAD/PDF/33799224.PDF. ACESSO EM: ABR. 2021.

ZATTERA, ADEMIR J. REUSO DE RESÍDUOS DE LAMINADOS DE FIBRA DE VIDRO NA CONSTRUÇÃO CIVIL. UNIVERSIDADE DE CAXIAS DO SUL, 200. DISPONÍVEL EM: HTTP://DOCPLAYER.COM.BR/21968916-REUSO-DE-RESIDUOSDE-LAMINADOS-DE-FIRA-DE-VIDRO-NA- CONSTRUCAO-CIVIL.HTML. ACESSO EM: ABR. 202I 